\title{
A Case-control Pilot Study on n-3 Polyunsaturated Fatty Acid as a Negative Risk Factor for Myocardial Infarction
}

\author{
Eiji OdA, ${ }^{1} \mathrm{MD}$, Katsuharu HATADA,${ }^{2} \mathrm{MD}$, Kiminori KATOH, ${ }^{2} \mathrm{MD}$, \\ Makoto KoDAMA, ${ }^{2} \mathrm{MD}$, Yuichi NAKAMURA, ${ }^{2} \mathrm{MD}$, \\ and Yoshifusa AIZAwA, ${ }^{2} \mathrm{MD}$
}

\begin{abstract}
SUMMARY
The relation between $\mathrm{n}-3$ polyunsaturated fatty acid (PUFA) and nonfatal myocardial infarction is still controversial.

A multicenter case-control pilot study on n-3 PUFA as a negative risk factor for myocardial infarction was performed in Niigata prefecture. Seventy-three patients with acute myocardial infarction (AMI) and age and gender matched controls $(n=84)$ were recruited. Serum leptin levels were significantly higher in patients with AMI than the controls $(8.1 \pm 6.7 \mathrm{ng} / \mathrm{mL}$ versus $5.8 \pm 3.7 \mathrm{ng} / \mathrm{mL}, P<0.01)$, and serum high-density lipoprotein cholesterol (HDLc) levels were significantly lower in patients with AMI than the controls $(46 \pm 10.5 \mathrm{mg} / \mathrm{dL}$ versus $60 \pm 15 \mathrm{mg} / \mathrm{dL}, P<0.00001)$. Statistically significant differences were preserved in leptin and HDLc when the data were analyzed separately by gender. Serum levels (\%weight) of linolenic acid (C18:3:n3), eicosapentaenoic acid (C20:5:n3), docosapentaenoic acid (C22:5:n3), and total n-3 PUFA were significantly lower in patients with AMI than the control group $(P<0.000001,<0.05,<0.05,<0.05$, respectively). The serum n-3 PUFA/saturated fatty acid (SF) ratio and n-3 PUFA/n-9 monounsaturated fatty acid (MUFA) ratio were significantly lower in patients with AMI than the controls $(P<0.05$ and $<0.01$, respectively). When the subjects were separated into two categories according to an n-3/n-6 PUFA ratio below 0.3 or above 0.3 , patients with AMI were more frequently in the former while the controls were more frequently in the latter $(P<0.05)$.

N-3 PUFA may be a negative risk factor for AMI. The results suggest leptin is a risk factor for AMI irrespective of ethnicity and gender. (Int Heart J 2005; 46: 583-591)
\end{abstract}

Key words: Myocardial infarction, Insulin resistance, Leptin, n-3 polyunsaturated fatty acid

N-3 PUFA has been proven to be a protective nutritional substance against sudden death. ${ }^{1,2)}$ However, the relationship between n-3 PUFA and nonfatal myocardial infarction is controversial. ${ }^{3-5)}$ Guallar, et al reported that there was no

From the ${ }^{1}$ Niigata Prefectural Yoshida Hospital, ${ }^{2}$ Division of Cardiology, Niigata University Graduate School of Medical and Dental Sciences, Niigata, Japan.

Address for correspondence: Eiji Oda, MD, Niigata Prefectural Yoshida Hospital, Daibochou 32-14, Yoshida, Nishikanbara, Niigata 959-0242, Japan.

Received for publication December 10, 2004.

Revised and accepted February 24, 2005. 
difference in plasma fish oil levels between patients with myocardial infarction and controls in a prospective nested case-control study (the Physician's Health Study). ${ }^{3)}$

Soderberg, et al reported that the combination of high leptin and low apo A1 was associated with a particularly pronounced increased risk for AMI among male subjects in a region with a high incidence of cardiovascular disease. ${ }^{6)}$ Wallace, et al also reported in the large prospective West of Scotland Coronary Prevention Study (WOSCOPS) that leptin is a novel, independent risk factor for coronary heart disease in hypercholesterolemic males. ${ }^{7)}$

Leptin is a pleiotropic hormone ${ }^{8)}$ exerting diverse actions via its receptors in the hypothalamus and peripheral tissues. ${ }^{9)}$ Among these actions, recent interest has focused on its cardiovascular actions. Leptin stimulates vascular smooth muscle cell proliferation and migration, ${ }^{10)}$ enhances vascular calcification, ${ }^{11)}$ induces oxidative stress in endothelial cells, ${ }^{12,13)}$ induces hypertrophy in cultured cardiomyocytes, ${ }^{14)}$ and promotes vascular remodeling and neointimal growth, possibly, via its receptors in the intimal lesion. ${ }^{15)}$ Hyperleptinemia is associated with poor arterial compliance, ${ }^{16)}$ myocardial hypertrophy, ${ }^{17)}$ chronic heart failure, ${ }^{18)}$ essential hypertension, ${ }^{19)}$ and stroke. ${ }^{20)}$ Furthermore, there is a strong positive relationship between leptin and CRP, ${ }^{21)}$ implicating leptin as a factor that promotes vascular inflammation. These diverse actions of leptin suggest it is not only a novel risk factor, but also a major causal link for cardiovascular diseases, as is CRP. ${ }^{22,23)}$ Plasma leptin levels are reported to be elevated in nonobese persons with insulin resistance ${ }^{24)}$ which is related to metabolic syndrome, and the intracellular signaling pathways of insulin and leptin are interrelated. ${ }^{25)}$ Leptin resistance $^{26-31)}$ is a complex pathophysiological concept. However, it could be summarized as follows; 1) restricted transport capacity of the hormone across the blood-brain barrier, ${ }^{28)}$ 2) selective derangement of anorectic signals of the hormone in the hypothalamus preserving sympatho-excitatory pathways including hypertensive effects, ${ }^{29)}$ and 3) selective depression of pathways promoting fatty acid oxidation in the peripheral organs such as the liver ${ }^{27)}$ and skeletal muscle, ${ }^{30)}$ or selective resistance at the adipocyte level, ${ }^{31)}$ not ameliorating adverse cardiovascular effects including the direct atherogenic action of hyerleptinemia. ${ }^{15}$ Although there is no simple reliable clinical marker for insulin resistance, plasma leptin levels, which represent leptin resistance, ${ }^{26-31)}$ can be readily measured. In the current multicenter case-control pilot study on n-3 PUFA in Niigata prefecture, where the incidence of coronary disease is low, we measured serum total fatty acid composition and leptin levels, and found that n-3 PUFA and leptin may be risk factors for AMI. 


\section{Methods}

Subjects: The study was carried out in Niigata prefecture, which is located in central Japan. Patients with AMI ranging in age from 40 to 80 years old $(n=73)$ and age- and gender-matched control persons $(n=84)$ were recruited at the hospitals listed in the Appendix. Patients with diabetes mellitus (FBS > 140), an atherosclerotic cardiovascular disease (old myocardial infarction, angina pectoris, cerebrovascular disease, peripheral vascular disease) or a history of any of these diseases were excluded from the control group. Written informed consent was obtained from all subjects. The ethics committee of each hospital approved the study.

Measurements: Fasting blood samples were obtained from all subjects. Blood samples were obtained in the morning following admission for AMI. Body height and weight, blood pressure, and serum total cholesterol ( $\mathrm{T}$ chol), triglyceride (TG), and HDL cholesterol (HDLc) levels were measured at each hospital. Serum

Table I. Baseline Characteristics and Laboratory Data

\begin{tabular}{lccccc}
\hline & AMI & $\mathrm{n}_{1}$ & Control & $\mathrm{n}_{2}$ & $P$ \\
\hline Age & $65 \pm 10$ & 73 & $64 \pm 10$ & 84 & $\mathrm{NS}$ \\
Age M & $64 \pm 10$ & 61 & $62 \pm 10$ & 61 & $\mathrm{NS}$ \\
Age F & $65 \pm 11$ & 12 & $64 \pm 9$ & 23 & $\mathrm{NS}$ \\
Smokers & 48 & 73 & 30 & 72 & $<0.01$ \\
SmokersM & 46 & 61 & 28 & 53 & $<0.01$ \\
SmokersF & 2 & 12 & 2 & 19 & $\mathrm{NS}$ \\
BMI & $24.8 \pm 3.4$ & 68 & $23.9 \pm 3.9$ & 66 & $\mathrm{NS}$ \\
BMI M & $24.5 \pm 3.1$ & 58 & $24.5 \pm 3.9$ & 46 & $\mathrm{NS}$ \\
BMI F & $26.4 \pm 4.2$ & 10 & $22.7 \pm 3.8$ & 20 & $\mathrm{NS}$ \\
Tchol & $189 \pm 33$ & 67 & $195 \pm 37$ & 72 & $\mathrm{NS}$ \\
Tchol M & $188 \pm 32$ & 57 & $191 \pm 32$ & 51 & $\mathrm{NS}$ \\
Tchol F & $195 \pm 41$ & 10 & $203 \pm 45$ & 21 & $\mathrm{NS}$ \\
TPEG & $118 \pm 64$ & 67 & $119 \pm 72$ & 70 & $\mathrm{NS}$ \\
TG M & $120 \pm 67$ & 57 & $121 \pm 77$ & 50 & $\mathrm{NS}$ \\
TG F & $103 \pm 48$ & 10 & $113 \pm 60$ & 20 & $\mathrm{NS}$ \\
HDLc & $46.0 \pm 10.5$ & 67 & $59.5 \pm 15.3$ & 65 & $<0.00001$ \\
HDLc M & $44.4 \pm 9.8$ & 57 & $56.2 \pm 14.3$ & 46 & $<0.00001$ \\
HDLc F & $54.7 \pm 10.9$ & 10 & $67.3 \pm 15.2$ & 19 & $<0.05$ \\
Leptin & $8.1 \pm 6.7$ & 72 & $5.8 \pm 3.7$ & 84 & $<0.01$ \\
Leptin M & $6.6 \pm 4.5$ & 60 & $5.0 \pm 2.6$ & 61 & $<0.05$ \\
Leptin F & $15.8 \pm 10.4$ & 12 & $7.9 \pm 5.2$ & 23 & $<0.05$ \\
& & & &
\end{tabular}

Data are presented as the mean \pm SD. M represents male and F represents female. $\mathrm{n} 1$ and $\mathrm{n} 2$ are the number of values obtained from the AMI and control groups, respectively. $P$ values were calculated using an unpaired $t$ test. T chol (mg/dL), TG (mg/dL), HDLc (mg/dL), leptin ( $\mathrm{ng} / \mathrm{mL})$. 
Table II. Serum Total Fatty Acid Composition

\begin{tabular}{|c|c|c|c|}
\hline \%weight & AMI & Control & $P$ \\
\hline $\mathrm{C} 12: 0$ & $0.04 \pm 0.04$ & $0.07 \pm 0.09$ & $<0.01$ \\
\hline C14:0 & $0.69 \pm 0.23$ & $0.96 \pm 0.40$ & $<0.000001$ \\
\hline $\mathrm{C} 16: 0$ & $24.56 \pm 1.41$ & $23.53 \pm 2.31$ & $<0.001$ \\
\hline C16:1:n7 & $2.21 \pm 0.71$ & $2.24 \pm 0.81$ & NS \\
\hline C18:0 & $6.74 \pm 0.57$ & $7.18 \pm 0.54$ & $<0.00001$ \\
\hline C18:1:n9 & $20.35 \pm 2.23$ & $19.48 \pm 2.69$ & $<0.05$ \\
\hline C18:2:n6 & $26.59 \pm 3.21$ & $26.94 \pm 3.91$ & NS \\
\hline C18:3:n6 & $0.28 \pm 0.14$ & $0.32 \pm 0.17$ & NS \\
\hline C18:3:n3 & $0.68 \pm 0.18$ & $0.86 \pm 0.25$ & $<0.000001$ \\
\hline C20:0 & $0.26 \pm 0.04$ & $0.24 \pm 0.04$ & $<0.05$ \\
\hline C20:1:n9 & $0.17 \pm 0.04$ & $0.20 \pm 0.07$ & $<0.05$ \\
\hline C20:2:n6 & $0.19 \pm 0.03$ & $0.19 \pm 0.03$ & NS \\
\hline C20:3:n6 & $1.12 \pm 0.33$ & $0.98 \pm 0.29$ & $<0.01$ \\
\hline C20:4:n6 & $4.90 \pm 1.04$ & $4.86 \pm 0.98$ & NS \\
\hline C20:5:n3 & $2.86 \pm 1.43$ & $3.41 \pm 1.90$ & $<0.05$ \\
\hline C22:0 & $0.61 \pm 0.11$ & $0.56 \pm 0.13$ & $<0.005$ \\
\hline $\mathrm{C} 22: 4: \mathrm{n} 6$ & $0.11 \pm 0.03$ & $0.12 \pm 0.03$ & NS \\
\hline $\mathrm{C} 22: 5: \mathrm{n} 3$ & $0.72 \pm 0.18$ & $0.78 \pm 0.19$ & $<0.05$ \\
\hline $\mathrm{C} 22: 6: \mathrm{n} 3$ & $4.91 \pm 1.16$ & $5.17 \pm 1.37$ & NS \\
\hline C24:0 & $0.55 \pm 0.10$ & $0.52 \pm 0.10$ & NS \\
\hline C24:1:n9 & $1.33 \pm 0.31$ & $1.25 \pm 0.35$ & NS \\
\hline$\Sigma \mathrm{SF}$ & $33.43 \pm 1.45$ & $33.05 \pm 2.44$ & NS \\
\hline$\Sigma$ n9 MUFA & $21.86 \pm 2.10$ & $20.93 \pm 2.57$ & $<0.05$ \\
\hline इn6 PUFA & $33.20 \pm 3.44$ & $33.41 \pm 4.18$ & NS \\
\hline$\Sigma$ n3 PUFA & $9.16 \pm 2.43$ & $10.2 \pm 3.31$ & $<0.05$ \\
\hline$\Sigma \mathrm{n} 3 / \Sigma \mathrm{SF}$ & $0.27 \pm 0.08$ & $0.31 \pm 0.11$ & $<0.05$ \\
\hline$\Sigma \mathrm{n} 3 / \Sigma \mathrm{n} 9$ & $0.43 \pm 0.15$ & $0.51 \pm 0.22$ & $<0.01$ \\
\hline$\Sigma \mathrm{n} 3 / \Sigma \mathrm{n} 6$ & $0.28 \pm 0.09$ & $0.32 \pm 0.13$ & NS \\
\hline
\end{tabular}

Data are presented as the mean \pm SD. SF represents saturated fatty acid, MUFA monounsaturated fatty acid, and PUFA polyunsaturated fatty acid.

total fatty acids, leptin, and high-sensitivity CRP were measured by a commercial laboratory in Japan (SRL Inc.). Serum total fatty acids were measured using Omegawax 250 (SUPELCO, gas-chromatography), leptin using HUMAN LEPTIN RIA KIT (LINCO Research Inc.), and high-sensitivity CRP using N-latex CRP (DADE BEHRING). Smoking history was obtained from each person.

Statistical analysis:The numerical values are presented as the mean \pm SD. Group comparisons were conducted using an unpaired $t$ test. Categorical variables were compared using the $\chi^{2}$ test. Correlations were analyzed using Pearson's product moment correlation coefficients and Fisher's z-transformation. $P$ values less than 


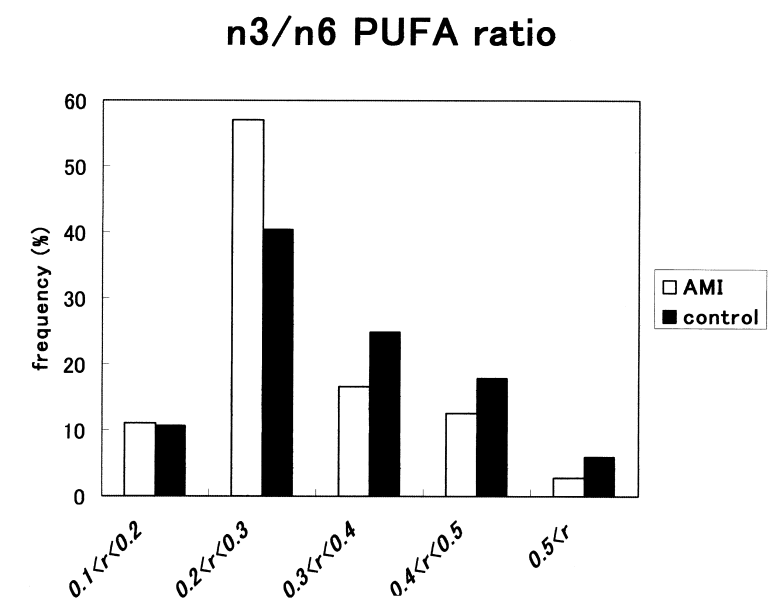

Figure: Distribution of n-3/n-6 PUFA ratio in AMI and control groups. An n-3/ n-6 PUFA ratio higher than 0.3 favored the control group $\left(P<0.05 \chi^{2}\right.$ test). $\mathrm{r}=\mathrm{n}$ 3/n-6 PUFA ratio.

0.05 were considered to be significant. The relative significances of BMI, leptin, TG, T chol, and HDLc were examined by logistic regression analysis.

\section{Results}

Clinical data: The baseline characteristics and laboratory data of the patients and control subjects are presented in Table I. There were no statistically significant differences in BMI, TG, or T chol between the cases and controls.

Leptin and HDLc: Significant differences were found in leptin and HDLc between the two groups (Table I). The difference in serum leptin was significant when males and females were analyzed separately (Table I). Although leptin and BMI were closely correlated $(P<0.0001)$, there was no significant difference in BMI between the cases and controls $(P=0.173)$. In logistic regression analysis, BMI, TG, and T chol were discarded as independent variables $(\beta=-0.117,-0.004$, -0.00015 , respectively, and $P=0.127,0.308,0.985$, respectively), though HDLc and leptin were adopted as independent variables $(\beta=-0.099$ and +0.132 , respectively, and $P=0.00003$ and 0.037 , respectively).

Fatty acid composition: Serum levels (weight\%) of lauric acid (C12:0), myristic acid (C14:0), stearic acid (C18:0), linolenic acid (C18:3:n3), eicosenic acid (C20:1:n9), eicosapentaenoic acid (EPA, C20:5:n3), docosapentaenoic acid (C22:5:n3), and total n-3 PUFA were significantly lower, and those of palmitic 
acid (C16:0), oleic acid (C18:1:n9), arachidic acid (C20:0), and dihomo- $\gamma$-linolenic acid (C20:3:n6) were significantly higher in patients with AMI than the control group (Table II). The serum n-3 PUFA/saturated fatty acid (SF) ratio and n-3 PUFA/n-9 monounsaturated fatty acid (MUFA) ratio were significantly lower in patients with AMI than the controls (Table II, $P<0.05$ and $<0.01$, respectively). When the subjects were divided into two categories according to the serum n-3/ n-6 PUFA ratio; those lower than 0.3 and those above 3, patients with AMI were more frequently in the former while the controls were more frequently in the latter (Figure 1, $P<0.05$ ).

\section{DISCUSSION}

Although this is a pilot study and the number of subjects is rather small, significant differences were found in serum levels of n-3 PUFA, leptin, and HDLc between the AMI patients and the control group. Even though the study populations were completely different, the present results are strikingly similar to those reported by Söderberg, et $a l^{6}$ in that the most pronounced differences between the AMI and control groups were seen in the levels of leptin and HDLc, though they measured apo A-1 instead of HDLc for a technical reason. Also, in the report by Wallace, et $a l,{ }^{7)}$ the differences in leptin $(P<0.0001)$ and HDLc $(P<0.0001)$ between the AMI and control groups were bigger than those in T chol (NS) and TG $(P<0.01)$. These previous two reports included no female subjects and were performed on populations in countries with high incidences of AMI. In contrast, the present study was performed in a population with a low incidence of AMI and included female subjects. When analyzed separately by gender, statistically significant differences in leptin and HDLc between patients with AMI and controls were also found among women. Therefore, leptin and HDLc may be strong risk factors for myocardial infarction irrespective of ethnicity and gender. However, our study is a cross sectional study and serum T chol, TG, HDLc, and leptin levels may be influenced by acute reactions following AMI. Therefore, further prospective studies are required.

Although protection against sudden death by n-3 PUFA has been established both among men with and without previous myocardial infarction (MI), ${ }^{1,2)}$ the relationship between n-3 PUFA and nonfatal MI is controversial. ${ }^{3-5)}$ In the present study, we found significantly lower n-3 PUFA levels in \%weight and lower n-3 PUFA/SF and n-3 PUFA/n-9 MUFA ratios in AMI subjects than in the controls in a population with higher n-3/n-6 PUFA ratios than those in Western countries, where the $n-3 / n-6$ PUFA ratio is approximately 0.1 . When the subjects were divided into two categories according to the serum n-3/n-6 PUFA ratio; less than 0.3 or greater than 0.3 ; patients with AMI were more frequently in the former 
while the controls were more frequently in the latter. From cross-sectional analysis of contemporary hunter-gatherer populations, it has been estimated that the n3/n-6 PUFA ratio in the human diet has been close to 1.0 during the 2-4 million years of human existence during which our genes adapted to the environment, including diet. ${ }^{32)}$ Therefore, it is possible that the optimal value of the $n-3 / n-6$ PUFA ratio may be higher than 0.3. Guallar, et al reported that there was no difference in plasma fish oil levels between patients with myocardial infarction and controls. ${ }^{3)}$ The n-3/n-6 PUFA ratio of their subjects may have been lower than the threshold. Winnicki, et al reported that a diet rich in fish is associated with lower plasma leptin, independent of body fat. ${ }^{33)}$ However, we were unable to verify an inverse correlation between leptin and the n-3/n-6 PUFA ratio.

Limitations: The present study was a cross-sectional case-control study, and serum levels of $\mathrm{T}$ chol, TG, HDLc, leptin, and fatty acids may have been influenced by acute reactions and prolonged fasting in the AMI group. Therefore, the present results may merely reflect the results of AMI, not the risks for AMI. However, our results are strikingly similar to the data reported by Soderberg, et $a l^{6}$ ) and Wallace, et al, ${ }^{7)}$ both of which were prospective nested case-control studies. Nevertheless, this concern should be resolved by further prospective studies.

Conclusion: The results of the present study indicate that relative serum levels of n-3 PUFA were significantly lower in patients with AMI than in controls and that the serum level of leptin was significantly higher in patients with AMI than in controls irrespective of gender. Serum n-3 PUFA levels may be a negative risk factor for AMI, however, further studies are still warranted.

\section{APPENDIX}

Investigators (Hospitals)

Yoshifusa Aizawa, Makoto Kodama, Haruo Hanawa, Kiminori Katoh, Yuichi Nakamura, Katsuharu Hatada (Niigata University Hospital); Kaoru Suzuki, Eiichi Itoh, Yasuhiko Tanabe, Akimitsu Nasuno (Niigata Prefectural Shibata Hospital); Takashi Tsuda, Takefumi Miyajima, Toshio Yamaguchi (Kido Hospital); Hideaki Ohtsuka, Yasushi Miyakita, Takaaki Shiono, Koutarou Higuchi (Niigata Kobari Hospital); Masaaki Okabe, Junji Ishiguro, Masahito Satoh, Hitoshi Kitazawa, Minoru Takahashi, Yoshio Ikeda, Satoru Hirono, Mitsuru Katoh (Tachikawa General Hospital); Tsuneo Nagai, Katsuya Ebe, Toshio Fujita, Yukiko Ohno (Nagaoka Red Cross Hospital); Fumiaki Masani, Osamu Ogawa, Hajime Takano (Niigata Prefectural Central Hospital); Kunio Kodera (Niigata Rousai Hospital); Minoru Murata, Kouji Oohira (Mito Saiseikai Hospital); Takanao Aoki, Takaaki Kubo, Ryuuta Imaki, Kazuyuki Ozaki (Takeda General Hospital); Akihiro Abe, Eiji Oda (Niigata Prefectural Yoshida Hospital). 


\section{ACKNOWLEDGMENTS}

We thank all subjects who participated in this study. This work was partially supported by Mochida Pharmaceutical Co., Ltd.

\section{REFERENCES}

1. Albert CM, Campos H, Stampfer M, et al. Blood levels of long-chain n-3 fatty acids and the risk of sudden death. N Engl J Med 2002; 346: 1113-8.

2. Marchioli R, Barzi F, Bomba E, et al. Early protection against sudden death by $n-3$ polyunsaturated fatty acids after myocardial infarction: time-course analysis of the results of the Gruppo Italiano per lo Studio della Sopravvivenza nell'Infarto Miocardico (GISSI)-Prevenzione. Circulation 2002; 105: 1897-903.

3. Guallar E, Hennekens CH, Sacks FM, Willett WC, Stampfer MJ. A prospective study of plasma fish oil levels and incidence of myocardial infarction in U.S. male physicians. J Am Coll Cardiol 1995; 25: 387-94.

4. Bucher HC, Hengstler P, Schindler C, Meier G N-3 polyunsaturated fatty acids in coronary heart disease: a meta-analysis of randomized controlled trials. Am J Med 2002; 112: 298-304.

5. Thies F, Garry JMC, Yaqoob P, et al. Association of n-3 polyunsaturated fatty acids with stability of atherosclerotic plaques: a randomized controlled trial. Lancet 2003; 361: 477-85.

6. Soderberg S, Ahren B, Jansson JH, et al. Leptin is associated with increased risk of myocardial infarction. J Intern Med 1999; 246: 409-18.

7. Wallace AM, McMahon AD, Packard CJ, et al. Plasma leptin and the risk of cardiovascular disease in the west of Scotland coronary prevention study (WOSCOPS). Circulation 2001; 104: 3052-6.

8. Zhang Y, Proenca R, Maffei M, Barone M, Leopold L, Friedman JM. Positional cloning of the mouse obese gene and its human homologue. Nature 1994; 372: 425-32.

9. Bjorbaek C, Kahn BB. Leptin signaling in the central nervous system and the periphery. Recent Prog Horm Res 2004; 59: 305-31. (Review)

10. Oda A, Taniguchi T, Yokoyama M. Leptin stimulates rat aortic smooth muscle cell proliferation and migration. Kobe J Med Sci 2001; 47: 141-50.

11. Parhami F, Tintut Y, Ballard A, Fogelman AM, Demer LL. Leptin enhances the calcification of vascular cells: artery wall as a target of leptin. Circ Res 2001; 88: 954-60.

12. Bouloumie A, Marumo T, Lafontan M, Busse R. Leptin induces oxidative stress in human endothelial cells. FASEB J 1999; 13: 1231-8.

13. Yamagishi S, Edelstein D, Du X, Kaneda Y, Guzman M, Brownlee M. Leptin induces mitochondrial superoxide production and monocyte chemoattractant protein-1 expression in aortic endothelial cells by increasing fatty acid oxidation via protein kinase A. J Biol Chem 2001; 276: 25096-100.

14. Xu FP, Chen MS, Wang YZ, et al. Leptin induces hypertrophy via endothelin-1-reactive oxygen species pathway in cultured neonatal rat cardiomyocytes. Circulation 2004; 110: 1269-75.

15. Schafer K, Halle M, Goeschen $\mathrm{C}$, et al. Leptin promotes vascular remodeling and neointimal growth in mice. Arterioscler Thromb Vasc Biol 2004; 24; 112-7.

16. Singhal A, Farooqi IS, Cole TJ, et al. Influence of leptin on arterial distensibility: a novel link between obesity and cardiovascular disease? Circulation 2002; 106: 1919-24.

17. Paolisso G, Tagliamonte MR, Galderisi M, et al. Plasma leptin level is associated with myocardial wall thickness in hypertensive insulin-resistant men. Hypertension 1999; 34: 1047-52.

18. Leyva F, Anker SD, Egerer K, Stevenson JC, Kox WJ, Coats AJ. Hyperleptinaemia in chronic heart failure. Relationships with insulin. Eur Heart J 1998; 19; 1547-51.

19. Agata J, Masuda A, Takada M, et al. High plasma immunoreactive leptin level in essential hypertension. Am J Hypertension 1997; 10: 1171-4.

20. Soderberg S, Stegmayr B, Ahlbeck-Glader C, Slunga-Birgander L, Ahren B, Olsson T. High leptin levels are associated with stroke. Cerebrovasc Dis 2003; 15: 63-9.

21. Shamsuzzaman ASM, Winnicki M, Wolk R, et al. Independent association between plasma leptin and C-reactive protein in healthy humans. Circulation 2004; 109; 2181-5. 
22. Ridker PM. High-sensitivity C-reactive protein: potential adjunct for global risk assessment in the primary prevention of cardiovascular disease. Circulation 2001; 103: 1813-8.

23. Yeh ET, Anderson HV, Pasceri V, Willerson JT. C-reactive protein: linking inflammation to cardiovascular complications. Circulation 2001; 104: 974-5.

24. Segal KR, Landt M, Klein S. Relationship between insulin sensitivity and plasma leptin concentration in lean and obese men. Diabetes 1996; 45: 988-91.

25. Zhao AZ, Shinohara MM, Huang D, et al. Leptin induces insulin-like signaling that antagonizes cAMP elevation by glucagons in hepatocytes. J Biol Chem 2000; 275: 11348-54.

26. Bjorbaek C, El-Haschimi K, Frantz JD, Flier JS. The role of SOCS-3 in leptin signaling and leptin resistance. J Biol Chem 1999; 274: 30059-65.

27. Huang W, Dedousis N, Bhatt BA, O'Doherty RM. Impaired activation of phosphatidylinositol 3-kinase by leptin is a novel mechanism of hepatic leptin resistance in diet-induced obesity. J Biol Chem 2004; 279: 21695700.

28. Caro JF, Kolaczynski JW, Nyce MR, et al. Decreased cerebrospinal-fluid/serum leptin ratio in obesity: a possible mechanism for leptin resistance. Lancet 1996; 348: 159-61.

29. Correia ML, Haynes WG, Rahmouni K, Morgan DA, Sivitz WI, Mark AL. The concept of selective leptin resistance: evidence from agouti yellow obese mice. Diabetes 2002; 51: 439-42.

30. Steinberg GR, Parolin ML, Heigenhauser GJ, Dyck DJ. Leptin increases FA oxidation in lean but not obese human skeletal muscle: evidence of peripheral leptin resistance. Am J Physiol 2002; 283: E187-92.

31. Huan JN, Li J, Han Y, Chen K, Wu N, Zhao AZ. Adipocyte-selective reduction of the leptin receptors induced by antisense RNA leads to increased adiposity, dyslipidemia, and insulin resistance. J Biol Chem 2003; 278 : 45638-50.

32. Leaf A, Weber PC. A new era for science in nutrition. Am J Clin Nutr 1987; 45: 1048-53.

33. Winnicki M, Somers VK, Accurso V, et al. Fish-rich diet, leptin, and body mass. Circulation 2002; 106: 28991. 